\title{
Cross Legume Species/Genera Transferability of SSR Markers and their Utility in Assessing Polymorphism among Advanced Breeding Lines in Dolichos Bean (Lablab purpureus L.)
}

\author{
M.S. Shivakumar ${ }^{1 *}$, S. Ramesh ${ }^{2}$, A. Mohan Rao ${ }^{2}$, H.R. Udaykumar ${ }^{2}$ and C.M. Keerthi ${ }^{2}$ \\ ${ }^{1}$ Indian Council of Agricultural Research-Indian Institute of Spices Research, \\ Kozhikode, Karnataka, India \\ ${ }^{2}$ Department of Genetics and Plant Breeding, University of Agricultural Sciences (UAS), \\ Bengaluru 560 065, Karnataka, India \\ *Corresponding author
}

\section{A B S T R A C T}

Keywords

Repeat motifs,

Transferability,

Legumes,

Cladogram.

Article Info

Accepted:

04 June 2017

Available Online:

10 August 2017
The use of transferable cross-species/genera SSR markers is an alternative strategy to ensure availability of markers in genomic resources-limited crops such as dolichos bean. A total of 275 cross legume species/genera SSR markers were examined for their transferability to dolichos bean. 126 of 275 cross legume species/genera SSR markers $(45.81 \%)$ were found transferable to dolichos bean. Transferability varied with cross legume species/genera with $83.33 \%$ of Medicago truncatula, $78.57 \%$ of greengram, $77.77 \%$ of chickpea, $53.33 \%$ of soybean and $33.33 \%$ of cowpea SSR markers being transferable to dolichos bean. The extent transferability of SSR markers based on simple di-/tri-nucleotide repeat motifs was higher than those based on penta-/tetra-/complex nucleotide repeat motifs. Of the 126 transferable SSR markers, 64 produced amplification in all the 14 advanced breeding lines with an average of 2.42 alleles per SSR locus. Six of the 64 SSR markers were most informative with four allelic variants.

\section{Introduction}

Dolichos bean (Lablab purpureus L Sweet) var. Lignosus is one of the most ancient legume crops known for its food (Ayyangar and Nambiar, 1935) and fodder (Magoon et al., 1974) values. In India, it is grown as a rain-fed crop for fresh pods containing immature grains for use as a vegetable and as a split dhal to a limited extent. The fresh pods are the harvestable economic product in dolichos bean. It is predominantly a selfpollinated crop (Ayyangar and Nambiar, 1935 ) with $2 n=2 x=22$ chromosomes (She and
Jiang, 2015) with a genome size of $367 \mathrm{Mbp}$ (Iwata et al., 2013). Handling of segregating generations derived from crosses involving deliberately selected parents followed by pedigree selection is the most widely used breeding method for genetic improvement of fresh pod yield and its component traits in dolichos bean (Keerthi et al., 2016).

Classical quantitative genetics-based breeding dolichos bean for pod productivity per se traits has been met with limited success, as 
these traits are controlled by a large number of genes with complex inheritance and significant cross-over genotype-byenvironment interaction. Therefore, phenotype-based selection of individuals for these traits has been less effective. DNA markers which are crop stage non-specific, environmental neutral, easily assayable and simply inherited are increasingly being used as surrogates of difficult-to-breed productivity per se traits (the activity popularly known as marker-assisted selection) to augment the pace and efficiency of breeding crop plants. However, identification of markers closely linked to genomic regions which explain substantial portion of phenotypic expression of productivity per se traits [the process is called as quantitative trait loci (QTL) mapping] is the pre-requisite for their use as surrogates. Of the several marker systems, those based on simple sequence repeats (SSR) are the primary choice of crop breeders owing to their hyper variability, higher reproducibility, mono-locus multi-allelic and co-dominant inheritance, possibility of multiplexing and amenability for automation (Powell et al., 1996).

The use of DNA markers in general and SSR markers in particular in dolichos bean breeding is still in its infancy due to their nonavailability. There are no reported attempts of developing genomic/expressed sequence tags (EST) SSR markers in dolichos bean. De novo development of SSR markers is expensive, laborious and labour intensive (Powell et al., 1996). Nevertheless, the use of transferable cross-species/genera SSR markers in crops where they are not available is an alternative strategy to ensure availability of markers in genomic resources-limited crops such as dolichos bean. Examination of transferability of cross legume species/genera SSR markers in dolichos bean is a prerequisite for their use as surrogates of difficult-to-breed traits. The discovery of high degree of genome synteny among fabaceae members such as soybean, cowpea, mung bean, common bean and alfalfa (Humphry et al., 2002; Choumane et al., 2004) offers opportunity to transfer SSR markers from these crops to genomic resources-limited crops like dolichos bean, a member of fabaceae. However, such studies are limited in dolichos bean. The only reported attempt to examine transferability of cross legume species/genera SSR markers to dolichos bean is from soybean (Yao et al., 2012). The objectives of the present study are to (1) explore transferability of SSR markers from a few other cross legume species/genera such as cowpea, Medicago truncatula, greengram and chickpea including soybean to dolichos bean and (2) assess the utility of transferable SSR markersto detect and characterize polymorphism at SSR loci among dolichos bean advanced breeding lines (ABL) differing for pod yield and its component traits (Keerthi et al., 2016).

\section{Materials and Methods}

A pair of dolichos bean genotypes (HA 4 and Kadlavare) differing in pod yield and its component traits were used for examining the transferability of cross legume species/genera SSR markers. While HA 4 is a highly popular high yielding photoperiod insensitive determinate bred variety, kadalavare is a high yielding photoperiod sensitive indeterminate landrace variety (Vaijayanthi et al., 2016). The total genomic DNA was extracted from 20 days old seedlings of these two varieties using the Cetyl Trimethyl Ammonium Bromide method (Doyle and Doyle, 1987). The quality and quantity of extracted genomic DNA was checked using $0.8 \%$ agarose gel by comparing with uncut lambda DNA.

A total of 275 cross legume species/genera EST and genomic SSR markers which included $\quad 150$ from cowpea 
(http://cowpeagenomics.med.virginia.edu/CG KB/), 90 from soybean (Peakell et al., 1998; Gupta and Prasad, 2009) (http://www.soybase.org/BARCSOYSSR/ind ex.php), 12 from Medicago truncatula (Gupta et al., 2012), 14 from greengram (Choudhary et al., 2009) and 9 from chickpea (Bostein et al., 1980) were used to amplify genomic DNA extracted from the selected pair of dolichos bean varieties.

\section{SSR marker assay}

The SSR priming regions of the two varieties were amplified using polymerase chain reaction (PCR) with Taq DNA polymerase. PCR mixtures contained approximately $2.0 \mu \mathrm{l}$ of DNA (30ng per $\mu \mathrm{l}$ ), $0.3 \mu \mathrm{Taq}$ polymerase (1 unit per $\mu \mathrm{l}$ ), $1.0 \mu \mathrm{l} 10 \mathrm{X}$ TE buffer, $0.5 \mu \mathrm{l}$ DNTPs $(2 \mathrm{mM})$ and $1.0 \mu \mathrm{l}$ each of forward and reverse primers $(1 \mu \mathrm{M})$ in a total of $10 \mu \mathrm{l}$ solution. The PCR cycle consisted of $5 \mathrm{~min}$ at $95^{\circ} \mathrm{C}$ (hot start), $0.30 \mathrm{~min}$ at $95^{\circ} \mathrm{C}$ (denaturation), $1 \mathrm{~min}$ at different annealing temperature, $1 \mathrm{~min}$ at $72^{\circ} \mathrm{C}$ (extension), 10 min at $72^{\circ} \mathrm{C}$ (final extension) followed by infinite time at $4^{\circ} \mathrm{C}$ for holding. The denaturation, annealing and extension step were carried out for 40 cycles. The PCR products were loaded on two per cent sigma agrose gel in $1 \mathrm{X}$ TAE buffer stained with ethidium bromide and bromophenol blue as loading dye. Amplicons were size separated using electrophoresis unit at $80 \mathrm{~V}$ for five hours using $1 \mathrm{X}$ TAE buffer.

\section{Criterion to assess transferability of SSR markers to dolichos bean}

Those SSR markers which successfully amplified SSR priming regions of DNA of the two genotypes and produced single and specific bands at reported expected product size range were considered as transferable SSR markers. Based on this criteria, per cent transferability was calculated as (Number of markers amplified/Total number of markers) $\times$ 100. A total of 126 cross legume species/genera SSR markers were transferable to dolichos bean. Per cent transferability of cross legume SSR markers classified by length (di/tri/tetra/penta/complex-nucleotides) repeat motifs was estimated. To take into account variable number of SSR markers from different crops and with different lengths of repeat motifs, conditional probability that a given transferable SSR marker was based on a particular legume crop/length of repeat motif was estimated as the ratio of number of transferable markers based on a particular legume crop/length of repeat motif to the total number of transferable markers.

\section{Assessment of genetic diversity at} transferable SSR marker loci

The material for this study consisted of 14 advanced breeding lines (ABL) differing in pod yield and its component traits (Keerthi et al., 2016). The genomic DNA was extracted from these $14 \mathrm{ABL}$ using the Cetyl Trimethyl Ammonium Bromide method (Doyle and Doyle, 1987) and quantified using $0.8 \%$ agarose gel. The $14 \mathrm{ABL}$ were genotyped using 126 transferable SSR markers. The transferable SSR markers' priming regions of 14 ABL were amplified following the procedure already described. The PCR products were loaded on four per cent sigma agrose gel in $1 \mathrm{X}$ TAE buffer stained with ethidium bromide and bromophenol blue as loading dye. Amplicons were size separated using electrophoresis unit at $80 \mathrm{~V}$ for five hours using $1 \mathrm{X}$ TAE buffer. Of the 126 transferable SSR markers, only 64 produced amplification in all the $14 \mathrm{ABL}$.

\section{Scoring of transferable SSR marker assay data}

The amplicons of 64 transferable SSR 
priming regions of genomic DNA at reported product size range (the amplicons in the same row) were scored as ' 1 ' for presence of amplicon and ' 0 ' for absence of amplicon for each of the transferable SSR marker locus. The variation in amplicon intensity was not taken into consideration to avoid ambiguity in scoring.

\section{Statistical analysis of SSR marker data}

The following population genetic parameters were estimated based on binary data of 64 transferable SSR markers which amplified in all the 14 ABL (supplementary 1).

Polymorphic SSR loci and major and minor allele frequencies

The per cent polymorphic loci were calculated as $\mathrm{kp} / \mathrm{k}$, where, ' $\mathrm{kp}$ ' is the number of polymorphic loci and ' $\mathrm{k}$ ' is the total number of SSR loci. The major alleles are those whose frequencies are $\leq 0.95$. Rare alleles are those whose frequencies are $\leq 0.05$.

\section{Polymorphic information content (PIC)}

The PIC, proposed as a measure of in formativeness of a genetic marker was estimated (Botstein et al., 1980) as

PIC $=1-\sum_{i=1}^{k} P_{i}^{2}-\sum_{i=1}^{k-1} \sum_{j=i+1}^{k} 2 P_{i}^{2} P_{I}^{2}$

Where $p_{i}$ is the frequency of the $i^{\text {th }}$ allele, $p_{j}$ is the frequency of the $j^{\text {th }}$ allele and ' $k$ ' is the number of alleles at transferable SSR loci.

\section{Nei's average gene diversity $\left(H_{e}\right)$}

The expected gene diversity for a transferable SSR locus locus was estimated (Nei, 1978) $\operatorname{ash}_{\mathrm{i}}=1-\mathrm{p}_{\mathrm{i}}^{2}-\mathrm{q}_{\mathrm{i}}^{2}$

Where, $p=$ frequency of $i^{\text {th }}$ allele and $q_{j}=$ frequency of $j^{\text {th }}$ allele
The average gene diversity $\left(\mathrm{H}_{\mathrm{e}}\right)$ across transferable SSR loci was estimated as

$$
\hat{\mathrm{H}}_{\mathrm{e}}=\sum_{\mathrm{i}=1}^{\mathrm{k}}\left(1-\mathrm{p}_{\mathrm{i}}^{2}-\mathrm{q}_{\mathrm{i}}^{2}\right)
$$

Where ' $\mathrm{k}$ ' is number of SSR loci

As sample size $(\mathrm{N})$ is small in the present study, an unbiased estimate of $\mathrm{H}_{\mathrm{e}}$ was estimated as

$$
\hat{\mathrm{H}}_{\mathrm{e}}=\frac{2 \mathrm{~N}}{(2 \mathrm{~N}-1)} \sum_{\mathrm{i}=1}^{\mathrm{k}}\left(1-\mathrm{p}_{\mathrm{i}}{ }^{2}-\mathrm{q}_{\mathrm{i}}{ }^{2}\right)
$$

Where, $\mathrm{N}=$ number of individuals (Nei, 1978).

\section{Average number of alleles per SSR locus} (n)

It was estimated by counting the number of detected alleles per SSR locus and averaging over ' $k$ ' SSR loci (Hartl and Clark, 1997) as indicated below.

$n=(1 / k) \sum_{i=1}^{k} n_{i}$,

Where, $\mathrm{n}_{\mathrm{i}}$ is the total number of detected alleles per SSR locus, and ' $\mathrm{k}$ ' is the number of SSR loci.

\section{Effective number of alleles $\left(A_{e}\right)$}

$\mathrm{A}_{\mathrm{e}}$, the effective number of alleles was calculated by inverting the measure of homozygosis at a locus.

$\mathrm{A}_{\mathrm{e}}=1 /(1-\mathrm{h})=1 / \sum_{i=1}^{k} \mathrm{p}_{\mathrm{i}}^{2}$

Where, $p_{i}$ is the frequency of the $i^{\text {th }}$ allele at a 
SSR locus and $\mathrm{h}=1-\Sigma \mathrm{p}_{\mathrm{i}}{ }^{2}$ is the estimate of heterozygosis at a SSR locus.

\section{Results and Discussion}

\section{Transferability of SSR markers}

One hundred and twenty six cross legume species/genera SSR markers (45.81\%) were found transferable to dolichos bean as they amplified SSR priming genomic regions of the two genetically diverse varieties. Transferability varied with cross legume species/genera with $83.33 \%$ of Medicago truncatula, $78.57 \%$ of greengram, $77.77 \%$ of chickpea, $53.33 \%$ of soybean and $33.33 \%$ of cowpea SSR markers being transferable to dolichos bean (Table 1; Fig. 1). The conditional probability that given cross species/genera SSR markers transferable to dolichos bean was higher from cowpea (closely followed by soybean) than those from other legume species/genera (Table 1; Fig. 2). Several researchers have also reported transferability of SSR markers among legume species/genera. For example (Chandra, 2011) has reported transferability of Medicago truncatula EST-SSR markers to forage legumes. (Gupta et al., 2012) have reported 92 per cent transferability of greengram SSR markers, 91 per cent of adjuki bean SSR markers and 86 per cent of cowpea SSR markers to black gram. (Datta et al., 2013) reported highest transferability of SSR markers from common bean followed by lentil, field pea and chickpea to pigeonpea. (Agbagwa et al., 2015) reported successful transferability of SSR markers from Vigna savi to pigeonpea. The transferability of cDNA SSR markers from Vicia sativa sub sp. sativa to $V$. ervilia and $V$. sativa sub sp. nigra ranged from $33 \%$ to $82 \%$, respectively with an average of 52\% (Raveendar et al., 2015).

The extent of transferability of SSR markers based on simple di-/tri-nucleotide repeat motifs was higher than those based on penta-/ tetra-/complex nucleotide repeat motifs (Table 2; Figs. 3 and 4). The present study provided evidence for transferability of cross legume species/genera SSR markers with high transferability success from cowpea and soybean to dolichos bean. The study suggested that it is preferable to choose and use simple di-/tri-nucleotide repeat motifsbased SSR markers for studies aimed at transferability of crops legume species/genera SSR markers to dolichos bean. The success of transferability of SSR markers developed in one species/genera to other species/genera could be attributed to high degree of DNA sequence conservation and stability of primer binding sites flanking SSR regions during evolution of legume species/genera (Decrooq et al., 2003; Zucchi et al., 2003). Among the legumes, a high level of genome conservation between cowpea and mung bean, mung bean and common bean and mung bean and dolichos bean has been (Humphry et al., 2002).

The present study help enrich the available SSR markers in dolichos bean. The 64 transferable SSR markers which produced successful amplification were used to assess polymorphism among ABL differing in pod yield and its component traits (Keerthi et al., 2016).

Transferable SSR markers assay-based polymorphism among $\mathrm{ABL}$

\section{Population genetic parameters}

The 64 transferable SSR markers used in the present study were polymorphic with an average of 2.42 alleles per SSR locus while four SSR markers (M 11, M 55 and M 55) were monomorphic (least informative) (Table 3). Six of the 64 SSR markers M 4, M 6, M 9, M 21, M 22 and M 39 were most informative with four allelic variants. 
Fig.1 Per cent transferability of cross legume crop species/genera SSR markers to dolichos bean

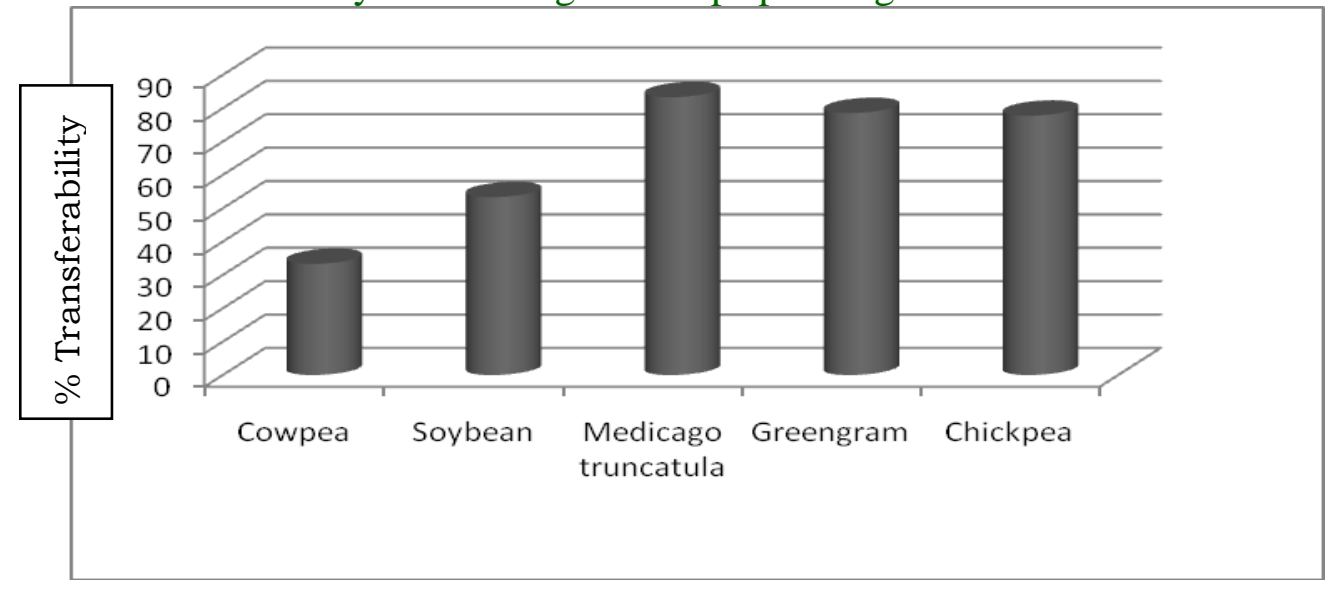

Fig.2 Conditional probability that a given cross legume species/genera SSR marker transferable to dolichos bean is from a particular crop

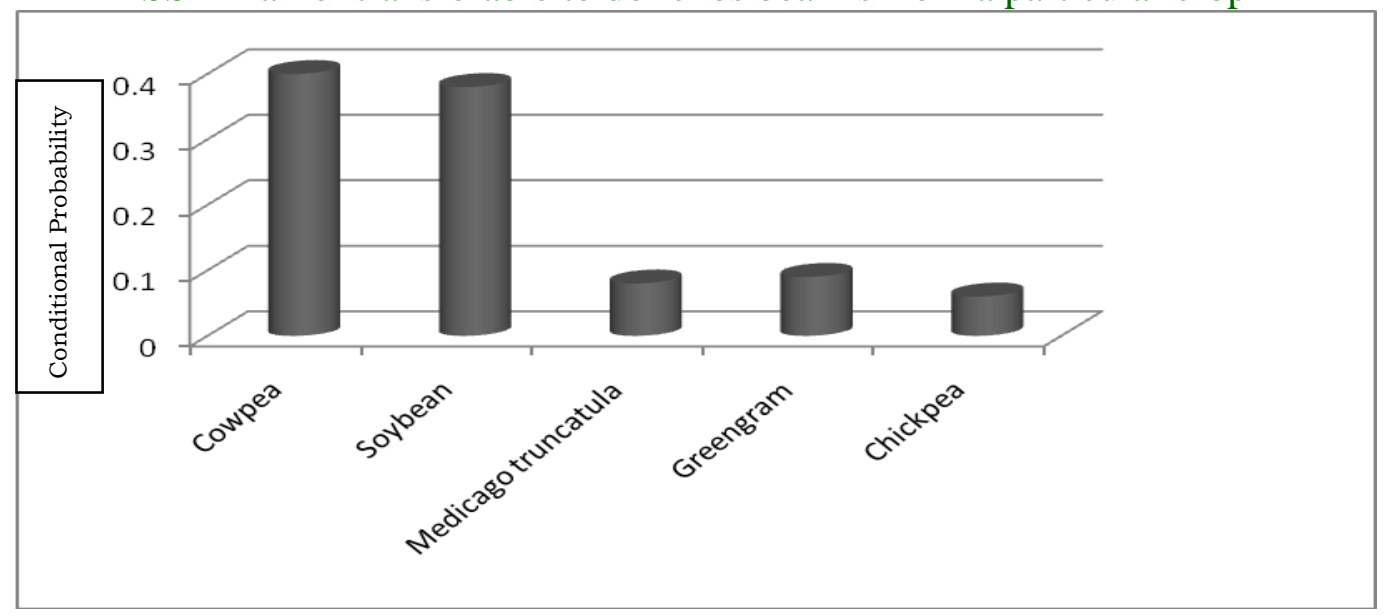

Fig.3 Per cent transferability of cross legume species/genera SSR markers (classified by length of repeat motifs) to dolichos bean

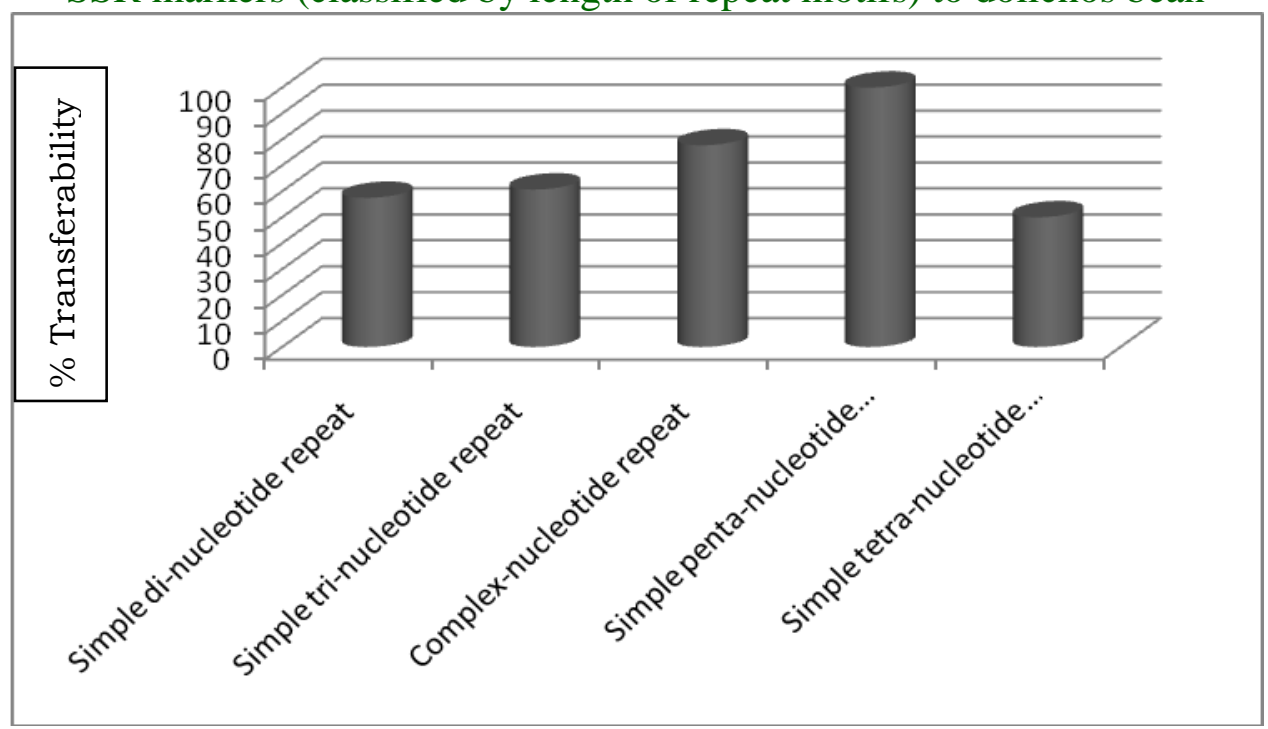


Fig.4 Conditional probability that a given cross legume species/genera SSR markers (classified by length of repeat motifs) transferable to dolichos bean

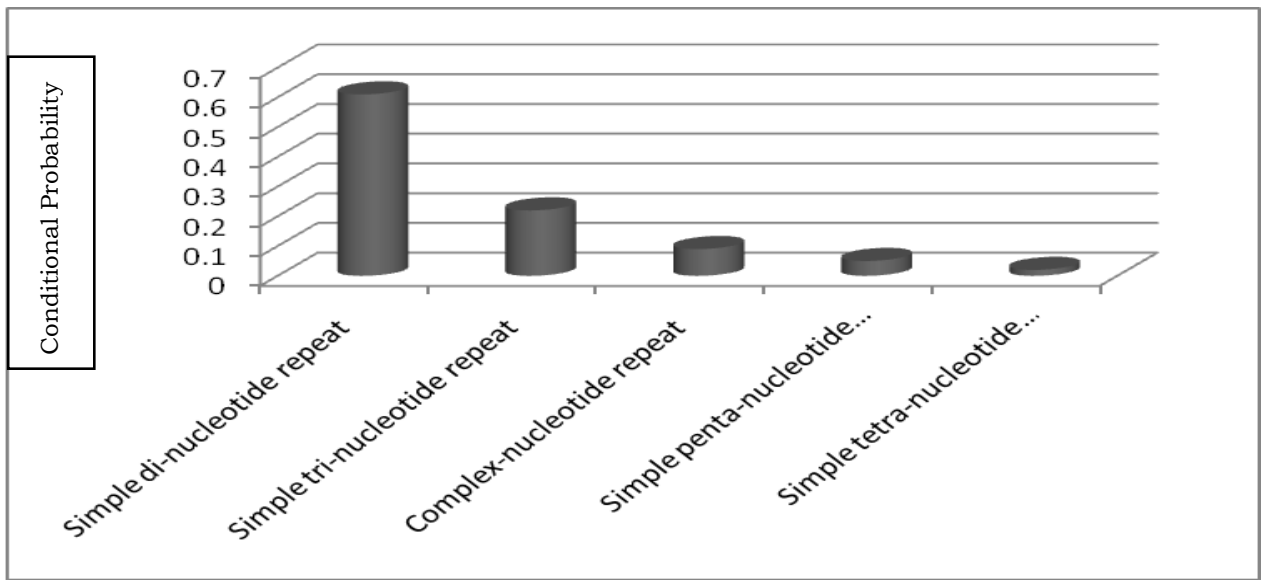

Fig.5 Cladogram depicting classification of 14 dolichos bean advanced breeding lines based on Transferable cross legume species/genera SSR marker allele frequencies

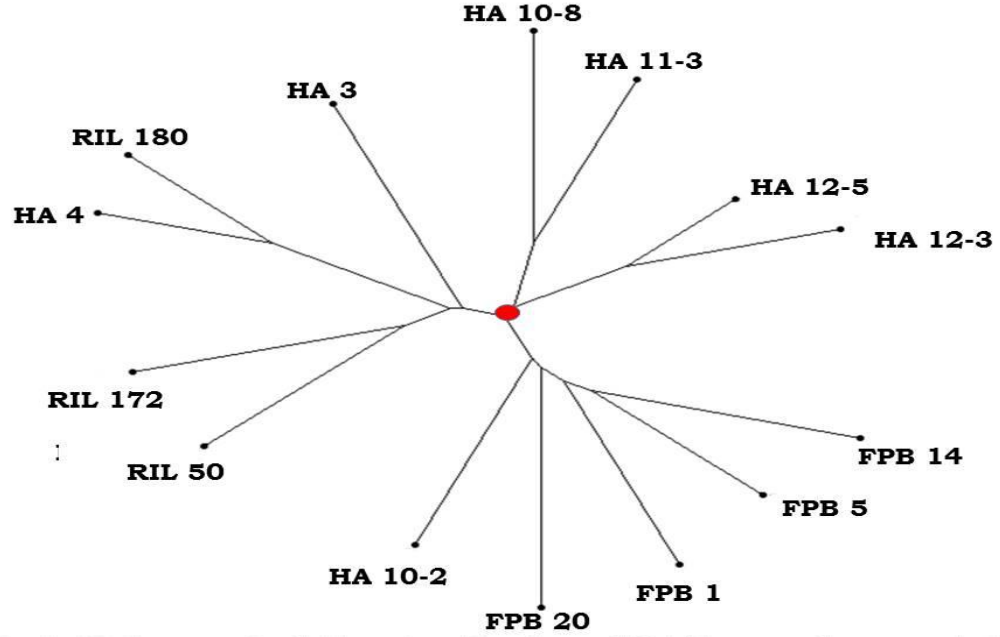

Table.1 Per cent transferability of cross legume species/genera SSR markers to dolichos bean

\begin{tabular}{ccccc}
\hline Crop & $\begin{array}{c}\text { Total number } \\
\text { of SSR } \\
\text { markers used }\end{array}$ & $\begin{array}{c}\text { Number of } \\
\text { SSR markers } \\
\text { amplified }\end{array}$ & \% Transferability & $\begin{array}{c}\text { Conditional probability } \\
\text { that a given cross } \\
\text { legume species/genera } \\
\text { SSR markers } \\
\text { transferable to dolichos } \\
\text { bean is from a } \\
\text { particular crop }\end{array}$ \\
\hline Cowpea & 150 & 50 & & 0.40 \\
Soybean & 90 & 48 & 33.33 & 0.38 \\
Medicago & 12 & 10 & 53.33 & 0.08 \\
truncatula & 14 & 11 & 83.33 & 0.09 \\
Greengram & 09 & 07 & 78.57 & 0.06 \\
Chickpea & $\mathbf{2 7 5}$ & $\mathbf{1 2 6}$ & 77.77 & \\
\hline Total & & $\mathbf{4 5 . 8 1}$ & \\
\hline
\end{tabular}


Table.2 Per cent transferability of cross legume species/genera SSR markers classified by length of repeat motifs to dolichos bean

\begin{tabular}{lcccc}
\hline $\begin{array}{l}\text { Length of repeat } \\
\text { motifs of cross } \\
\text { legume }\end{array}$ & $\begin{array}{l}\text { Total number of } \\
\text { SSR markers } \\
\text { usecies/genera SSR } \\
\text { markers }\end{array}$ & $\begin{array}{l}\text { Number of SSR } \\
\text { markers amplified }\end{array}$ & \% Transferability & $\begin{array}{l}\text { Conditional probability that } \\
\text { a given transferable cross } \\
\text { legume species/genera SSR } \\
\text { marker is based on a } \\
\text { particular motif }\end{array}$ \\
\hline $\begin{array}{l}\text { Simple di-nucleotide } \\
\text { repeat }\end{array}$ & 80 & 46 & 57.50 & 0.61 \\
$\begin{array}{l}\text { Simple tri-nucleotide } \\
\text { repeat }\end{array}$ & 28 & 17 & 60.71 & 0.22 \\
$\begin{array}{l}\text { Complex-nucleotide } \\
\text { repeat }\end{array}$ & 09 & 07 & 77.77 & 0.09 \\
$\begin{array}{l}\text { Simple penta- } \\
\text { nucleotide repeat }\end{array}$ & 04 & 04 & 100.00 & 0.05 \\
$\begin{array}{l}\text { Simple tetra- } \\
\text { nucleotide repeat }\end{array}$ & 04 & 02 & 50.00 & 0.02 \\
\hline
\end{tabular}

Table.3 Estimates of parameters specifying transferable cross legume species/genera SSR marker alleles-based diversity among advanced breeding lines of dolichos bean

\begin{tabular}{|c|c|c|c|c|c|c|c|c|c|c|c|c|c|}
\hline $\begin{array}{c}\text { SSR } \\
\text { Locus }\end{array}$ & $\mathrm{Na}^{*}$ & Maj* & Min* & $\mathrm{Ne}^{*}$ & $\mathrm{H}^{*}$ & PIC & $\begin{array}{c}\text { SSR } \\
\text { Locus }\end{array}$ & $\mathrm{Na}^{*}$ & Maj* & $\operatorname{Min} *$ & $\mathrm{Ne}^{*}$ & $\mathrm{H}^{*}$ & PIC \\
\hline M01 & 3.00 & 0.50 & 0.10 & 2.38 & 0.58 & 0.65 & M33 & 2.00 & 0.63 & 0.37 & 1.88 & 0.47 & 0.57 \\
\hline M02 & 2.00 & 0.70 & 0.30 & 1.72 & 0.42 & 0.55 & M34 & 2.00 & 0.60 & 0.40 & 1.92 & 0.48 & 0.58 \\
\hline M03 & 3.00 & 0.45 & 0.09 & 2.37 & 0.58 & 0.63 & M35 & 3.00 & 0.43 & 0.21 & 2.80 & 0.64 & 0.57 \\
\hline M04 & 4.00 & 0.71 & 0.07 & 1.85 & 0.46 & 0.43 & M36 & 3.00 & 0.64 & 0.09 & 2.05 & 0.51 & 0.60 \\
\hline M05 & 2.00 & 0.63 & 0.36 & 1.86 & 0.46 & 0.55 & M37 & 2.00 & 0.67 & 0.33 & 1.80 & 0.44 & 0.50 \\
\hline M06 & 4.00 & 0.61 & 0.07 & 2.25 & 0.56 & 0.57 & M38 & 3.00 & 0.67 & 0.08 & 1.95 & 0.49 & 0.55 \\
\hline M07 & 2.00 & 0.70 & 0.30 & 1.72 & 0.42 & 0.55 & M39 & 4.00 & 0.60 & 0.10 & 2.38 & 0.58 & 0.66 \\
\hline M08 & 3.00 & 0.71 & 0.07 & 1.78 & 0.44 & 0.39 & M40 & 2.00 & 0.64 & 0.36 & 1.86 & 0.46 & 0.55 \\
\hline M09 & 4.00 & 0.50 & 0.07 & 2.58 & 0.61 & 0.54 & M41 & 3.00 & 0.66 & 0.08 & 1.95 & 0.49 & 0.55 \\
\hline M10 & 2.00 & 0.56 & 0.44 & 1.98 & 0.49 & 0.59 & M42 & 2.00 & 0.71 & 0.29 & 1.69 & 0.41 & 0.32 \\
\hline M11 & 1.00 & 1.00 & 0.00 & 1.00 & 0.00 & 0.12 & M43 & 2.00 & 0.64 & 0.36 & 1.85 & 0.46 & 0.35 \\
\hline M12 & 3.00 & 0.66 & 0.08 & 1.95 & 0.49 & 0.55 & M44 & 2.00 & 0.57 & 0.43 & 1.96 & 0.49 & 0.37 \\
\hline M13 & 2.00 & 0.78 & 0.22 & 1.51 & 0.34 & 0.28 & M45 & 2.00 & 0.57 & 0.43 & 1.96 & 0.49 & 0.37 \\
\hline M14 & 3.00 & 0.64 & 0.28 & 2.00 & 0.50 & 0.43 & M46 & 3.00 & 0.54 & 0.07 & 2.25 & 0.56 & 0.54 \\
\hline M15 & 2.00 & 0.60 & 0.40 & 1.92 & 0.48 & 0.58 & M47 & 3.00 & 0.71 & 0.07 & 1.78 & 0.44 & 0.39 \\
\hline M16 & 2.00 & 0.78 & 0.22 & 1.51 & 0.34 & 0.28 & M48 & 2.00 & 0.82 & 0.18 & 1.42 & 0.30 & 0.46 \\
\hline M17 & 2.00 & 0.57 & 0.43 & 1.96 & 0.49 & 0.37 & M49 & 2.00 & 0.67 & 0.33 & 1.80 & 0.44 & 0.57 \\
\hline M18 & 2.00 & 0.82 & 0.18 & 1.42 & 0.30 & 0.46 & M50 & 2.00 & 0.91 & 0.09 & 1.20 & 0.17 & 0.39 \\
\hline M19 & 2.00 & 0.42 & 0.58 & 1.95 & 0.49 & 0.52 & M51 & 2.00 & 0.70 & 0.30 & 1.72 & 0.42 & 0.55 \\
\hline M20 & 2.00 & 0.6 & 0.4 & 1.92 & 0.48 & 0.58 & M52 & 2.00 & 0.71 & 0.29 & 1.69 & 0.41 & 0.32 \\
\hline M21 & 4.00 & 0.33 & 0.08 & 3.43 & 0.71 & 0.73 & M53 & 2.00 & 0.64 & 0.36 & 1.85 & 0.46 & 0.35 \\
\hline M22 & 4.00 & 0.41 & 0.08 & 3.13 & 0.68 & 0.70 & M54 & 2.00 & 0.86 & 0.14 & 1.32 & 0.24 & 0.21 \\
\hline M23 & 2.00 & 0.50 & 0.5 & 2.00 & 0.50 & 0.53 & M55 & 1.00 & 1.00 & 0.00 & 1.00 & 0.00 & 0.12 \\
\hline M24 & 3.00 & 0.50 & 0.21 & 2.65 & 0.62 & 0.55 & M56 & 2.00 & 0.86 & 0.14 & 1.32 & 0.24 & 0.21 \\
\hline M25 & 2.00 & 0.83 & 0.17 & 1.38 & 0.28 & 0.41 & M57 & 1.00 & 1.00 & 0.00 & 1.00 & 0.00 & 0.00 \\
\hline M26 & 3.00 & 0.50 & 0.21 & 2.65 & 0.62 & 0.55 & M58 & 2.00 & 0.67 & 0.33 & 1.80 & 0.44 & 0.50 \\
\hline M27 & 2.00 & 0.50 & 0.50 & 2.00 & 0.50 & 0.53 & M59 & 3.00 & 0.64 & 0.14 & 2.09 & 0.52 & 0.46 \\
\hline M28 & 3.00 & 0.75 & 0.12 & 1.68 & 0.41 & 0.55 & M60 & 3.00 & 0.64 & 0.14 & 2.09 & 0.52 & 0.46 \\
\hline M29 & 2.00 & 0.50 & 0.50 & 2.00 & 0.50 & 0.38 & M61 & 3.00 & 0.50 & 0.25 & 2.67 & 0.63 & 0.65 \\
\hline M30 & 2.00 & 0.70 & 0.30 & 1.72 & 0.42 & 0.55 & M62 & 3.00 & 0.50 & 0.21 & 2.65 & 0.62 & 0.55 \\
\hline M31 & 2.00 & 0.80 & 0.20 & 1.47 & 0.32 & 0.50 & M63 & 2.00 & 0.67 & 0.33 & 1.80 & 0.44 & 0.50 \\
\hline \multirow[t]{2}{*}{ M32 } & 2.00 & 0.86 & 0.14 & 1.32 & 0.24 & 0.21 & M64 & 2.00 & 0.64 & 0.36 & 1.85 & 0.46 & 0.35 \\
\hline & & & & & & & Mean & 2.42 & & & 1.91 & 0.44 & 0.47 \\
\hline
\end{tabular}

$* \mathrm{Na}=$ Observed number of alleles; $* \mathrm{Ne}=$ Effective number of alleles; ${ }^{*} \mathrm{H}=$ Nei's gene diversity; Maj = Major allele frequency; Min = Minor allele frequency; $\mathrm{PIC}=$ Polymorphic information content 
Supplementary.1 SSR primers used for cross-amplification in dolichos bean

\begin{tabular}{|c|c|c|c|c|c|}
\hline No. & Primer & Repeat motif & Forward primer sequence & Reverse primer sequence & Crop \\
\hline M1 & RAI9 & $*$ & CATGGAGATGAAGCTACAGATGGTG & GGTGCTTGCTCAACTAGATCTC & Cowpea \\
\hline M2 & AG2 & * & $\begin{array}{l}\text { ACGACGTTGTAAAAGTTTCACACACA } \\
\text { TTACTCTC }\end{array}$ & $\begin{array}{l}\text { CATTAAGTTCCCATTACCTCGG } \\
\text { CGATTGAGTGATTG }\end{array}$ & Cowpea \\
\hline M3 & Y101 & * & GTTTTGCTGACTAGAATGTTATTTTAC & GATTGTAGCTCCTTAGGAAG & Cowpea \\
\hline M4 & AG9 & * & $\begin{array}{l}\text { ACGACGTTGTAAAAGGTGGTAACAAT } \\
\text { TGGAGGGG }\end{array}$ & $\begin{array}{l}\text { CATTAAGTTCCCATTAGCTTGCA } \\
\text { ACTTCATTCACAG }\end{array}$ & Cowpea \\
\hline M5 & Y15 & * & GTCAGGCCACAACTACAAAG & CAGTCAACTCTTATTATCTATGACC & Cowpea \\
\hline M6 & Y67 & * & GAGAGTTGGATGGCAGATTTAAAAG & GCTTTAGAGGTGCAATCTTGGC & Cowpea \\
\hline M7 & CP267,СР268 & * & TCATGAGTTTCCACACACCAA & CCTTCGTATGTATATGTGGCTACTG & Cowpea \\
\hline M8 & MA87 & * & GACTACATCCAGCATTTGCACGAGC & GATGTTAGCAGTGATGATCTTCAGC & Cowpea \\
\hline M9 & Y20 & * & CTTCCGAGTTTTCCTTTATATAC & GGAATAGGTAGCAGAATAACATTTAG & Cowpea \\
\hline M10 & MA53 & * & GCAGAAGCGAAATCTCCTAGAAAGC & GAAAACAATGAACAAGGTGAGGTTC & Cowpea \\
\hline M11 & СР97,СР98 & * & TGCAATATAAAAACACTCTCGGATT & ATTTTGTGGCGACCTTTGAC & Cowpea \\
\hline M12 & CP337,СР338 & * & GGTGTCAACACCGTTGGAG & TGCAAGCCATTAGAGAATGACA & Cowpea \\
\hline M13 & EX24 & * & CGCTCCTCGCTGGCAAAAG & ССТTCCСТACAGTGATATTTCCC & Cowpea \\
\hline M14 & EX34 & * & GCTCACCTACGTGTGTTCGATC & GCAAGTGGATGTGGTGATCTC & Cowpea \\
\hline M15 & MS134 & * & GAACTTGATAGGATCCTAGA & TTCTGGTATGCACTGAGGGA & Cowpea \\
\hline M16 & EX 12 & * & CATGCGCCGTCAAAGAATACTG & GCATTCTTGATGTGTCTCTTACCTG & Cowpea \\
\hline M17 & EX39 & * & CAAGAGTCATTCGGCTCCTT & GCTGCACCGTTTTCCGAAAT & Cowpea \\
\hline M18 & MA83 & * & GAAGATACCAAGATGCCCCTAAAAC & GTATATGTTAGCTAGCCACGTATGA & Cowpea \\
\hline M19 & L-1 & * & GAATTCAACTCTCGTTTCTTCCG & GCATATATTTATATACACATACAAAC & Cowpea \\
\hline M20 & BSOYSSR_01_0646 & (AT) 20 & GGGCAAAATTTCATTTCCCT & TGAGTTGGAATTTATTTGGATGAA & Soybean \\
\hline M21 & BSOYSSR_01_1061 & (TA) 32 & GACTTGTGGTGTTGTTCTTCAA & TGAGCCAGCGTTAATCAAAA & Soybean \\
\hline M22 & BSOYSSR_01_1484 & (TC) 18 & СТTСТCTCAGCACССТCСАC & AАCССТTCTTCCACTTCCGT & Soybean \\
\hline M23 & BSOYSSR_04_0414 & (CT) 17 & CCATTCTACAATCATGCCCC & AGAAGCTGGCTAAGATGGCA & Soybean \\
\hline M24 & BSOYSSR_04_1136 & (AT) 21 & GAAATGACAATAATGCCGGG & TTCCATTCAAAGCAGAAGCA & Soybean \\
\hline M25 & BSOYSSR_05_0194 & (AT) 28 & CGTGATTTCCAATGTGCCTA & GCCACAACTAGCAAACACGA & Soybean \\
\hline M26 & BSOYSSR_05_1070 & (AT) 18 & TGGGTAGGTTTTTCAGCAATG & GCAAAGGGACCCAAAGGTAT & Soybean \\
\hline M27 & BSOYSSR_06_0026 & $(\mathrm{AG}) 18$ & AATTTGTAAGCAGCATGGCA & CTCTCCGTTCGTCACTCTCC & Soybean \\
\hline M28 & BSOYSSR_09_1284 & (CT) 18 & AGCGATGCAATTATTCCTGG & CATTTCCCATGTTTGGCTCT & Soybean \\
\hline M29 & BSOYSSR_14_1271 & (CT) 16 & AAGGAAGGAAAACCCATGCT & GGGACCACAGCGTTGAATTA & Soybean \\
\hline M30 & BSOYSSR_18_1580 & (TA) 19 & TGCCAAGAAACTATAGGCCG & TGAGTTTCCCTGGTAGTGTGG & Soybean \\
\hline M31 & BSOYSSR_18_1942 & (TC) 19 & CTCCTCATGCTTGGCAAAAT & ATGAGAACGCTGAAAAGGGA & Soybean \\
\hline M32 & BSOYSSR_20_1319 & (TA) 23 & GCGTAGAGCTGGGTTAGAGTAGTTA & GCGTAGAGCTGGGTTAGAGTAGTTA & Soybean \\
\hline
\end{tabular}




\begin{tabular}{|c|c|c|c|c|c|}
\hline No. & Primer & Repeat motif & Forward primer sequence & Reverse primer sequence & Crop \\
\hline M33 & BARCSOYSSR_20_1325 & (CT) 18 & GGAAGGAGGAAGGAACGAAG & AGAAAGAGCATTCGGGTGAA & Soybean \\
\hline M34 & BARCSOYSSR_20_1327 & (CT) 19 & TCATCTGCAGCACTGATTGA & TGGCTCCATAAGCACAAGAA & Soybean \\
\hline M35 & Satt526F & $(\mathrm{ATT}) 9$ & GCGGCAAATTCTAATGACTG & GTCGGAGTTCTCAGTC & Soybean \\
\hline M36 & Satt591F & (ATT) 17 & GCGCGACCTTAATGATA & GCGCCCAAAGCTTAAA & Soybean \\
\hline M37 & Satt534F & $(\mathrm{ATT}) 8$ & СТCCTCCTGCGCAACAACAATA & GGGGGATCTAGGCCATGAC & Soybean \\
\hline M38 & Satt547F & (ATT) 10 & GCGCTATCCGATCCATATGTG & TGATTTCGCTAGGTAAAATCA & Soybean \\
\hline M39 & Sat_369F & (AT) 28 & $\begin{array}{l}\text { GCGAATGGGGATAAACAATA } \\
\text { GAACAAGA }\end{array}$ & GCGCAGTGGCTTCACAT & Soybean \\
\hline M40 & Satt632F & $(\mathrm{ATT}) 17$ & GGGCTATGAAGGGAATGGAAAGGA & CCCATATTGAAGATTTG & Soybean \\
\hline M41 & Satt $233 \mathrm{~F}$ & (ATT) 16 & AAGCATACTCGTCGTAAC & GCGGTGCAAAGATA & Soybean \\
\hline M42 & Satt538F & $(\mathrm{ATT}) 12$ & GCAGGCTTATCTTAAGACAAGT & GGGGCGATAAACTAG & Soybean \\
\hline M43 & Gm000742F & (TC)5 & CTTCACAGAGAGAGGTGCCC & CTATTGGGTGGAAGGGTTGA & Soybean \\
\hline M44 & Gm001168F & (Tc) 16 & TGTGGTCCGATTGTTTGCTA & ACACCAAGCTCGAAAACCAC & Soybean \\
\hline M45 & Gm001362F & $(\mathrm{AG}) 5$ & ATCCACCGGTGTTGTGGTAT & GGTGGATCAAATGGTTGGAC & Soybean \\
\hline M46 & Gm000659F & $(\mathrm{GA}) 9$ & GATCATGGGCCAGCTTAAAA & AAACTGCTATGGGACCTCGT & Soybean \\
\hline M47 & Gm000664F & (TG)7 & GGTGCTGTTCGTGCTGTTAC & ACCGTCACAAAGCAAAAAGG & Soybean \\
\hline M48 & Mt_ESSR02F & $(\mathrm{CTTAT}) 4$ & GCATGCATTTTGTTGACCAC & GCCACCCAATAATCCAATGT & Medicago \\
\hline M49 & Mt_ESSR06F & $(\mathrm{CAT}) 5$ & AACTTCTGCGCAACGCTTAT & CGGAGAGCGTGAAAGAAGAG & Medicago \\
\hline M50 & Mt_ESSR39F & $(\mathrm{AAAG}) 5$ & AAATTTTCGGATGCGATGAG & TTAAAACACAACCGACACGC & Medicago \\
\hline M51 & VrSSR03F & (TGG)5 & AAGTTTTTGGTTGACCGCAG & CCCTTGCATAGACAGGTGGT & Vigna sps \\
\hline M52 & VrSSR04F & (CT) 5 & CTGATTCAGCCTCAGGTTCC & CACCGCTAAGATGCTCACAA & Vigna sps \\
\hline M53 & VrSSR12F & (AT)6-(AG)8 & ТСССТСТСССАССТТСТТСТ & GCTAGAGGGATGCTTCACCA & Vigna sps \\
\hline M54 & VrSSR13F & $(\mathrm{TG}) 7$ & TTGATACGGCCACTTTCTCC & CCATCAACGGTTTTTACGCT & Vigna sps \\
\hline M55 & VrSSR15F & $(\mathrm{AT}) 8$ & CATGACCGAGAAGACAAGCA & CCACAACAAATCCAAGAGCA & Vigna sps \\
\hline M56 & VrSSR16F & (TGTA)4 & TCTCCAТCСССАТСТTCATC & GGAGAGATCTGCGACCTTTG & Vigna sps \\
\hline M57 & VrSSR17F & $(\mathrm{TGTT}) 5$ & AACTTCGTCCTGCGCTTAAA & AGCATGACCACACCAATCAA & Vigna sps \\
\hline M58 & VrSSR19F & $(\mathrm{TA}) 5$ & AAATGTTCGTGGAATCCTGC & TTTCTTGTCCCTGAGTTCCAA & Vigna sps \\
\hline M59 & AG50B F & $*$ & ATAAATTGGAAGATGTGTTGGC & TACTGATGTGGATTCTCCCAA & Soybean \\
\hline M60 & AG93 F & $*$ & TCCATGCATGTATACTCCACC & TCATATGCCACAGGTTTTGTT & Soybean \\
\hline M61 & CESSRDB13F & $(\mathrm{AT}) 5$ & ATCTGGGAGCTTGTGAGTTA & TTGTATCTCCTTCAGATGGC & Chickpea \\
\hline M62 & CESSRDB18F & $\begin{array}{l}\text { (TA) } 5 \text { CA(TG) } 2 \\
\text { T(TG)3 }\end{array}$ & TGCAAATAAAGCCTTCAAGT & GAAAGTGGGAAAATGCAATA & Chickpea \\
\hline M63 & CESSRDB33F & $\begin{array}{l}\text { (GA)T(GA)TT } \\
(\mathrm{GA}) 5\end{array}$ & GCTGCACAAAAAGTACATGA & ATCCATCGAAACACCAATAG & Chickpea \\
\hline M64 & CESSRDB56F & (ATG) 4 & TGTCTGGAACAACAAGTGAG & GCCAATCAGATTTCCTCTTA & Chickpea \\
\hline
\end{tabular}


These six markers along with other 64 SSR markers were polymorphic with major allele frequency of $\leq 0.95$. The two tetra-allelic SSR markers (M 21 and M 22) and two tri-allelic markers (M 35 and M 61) exhibited greater ability to discriminate $14 \mathrm{ABL}$ as reflected by higher estimates of Nei's genetic diversity and PIC. These tri- and tetra allelic SSR markers were based on simple perfect di-/trinucleotide repeat motifs. The higher discriminating ability of tri- and tetra allelic SSR markers is expected as those markers with simple short perfect repeat motifs have higher mutation rates than those with long repeat motifs and hence exhibit greater allelic variability (Kelkar et al., 2008). Transferable SSR markers from Vigna savi revealed a total of 32 alleles in the range of 90-600 bp among 20 cultivars of cultivated species and ten genotypes of five wild species of pigeonpea (Agbagwa et al., 2015). In the present study, the estimates of PIC of 64 transferable SSR markers ranged from 0.12 to 0.66 among ABL of dolichos bean. The polymorphism of cross transferable SSR markers among legume crops has been amply demonstrated. The estimates of PIC of transferable SSR markers from Vigna savi to cultivated and wild relatives of Cajanus adams ranged from 0.24 to 0.69 with an average of 0.47 (Agbagwa et al., 2015). Datta et al., (2013) reported a fairly high estimates of PIC (ranging from 0.5 to 0.6 ) of cross transferable SSR markers from common bean, lentil, field pea and chickpea to pigeonpea. The reported significant differences in quantitative traits means of 14 ABL (Keerthi et al., 2016) were amply reflected at transferable SSR marker allelic diversity in the present study. The number of alleles needed to provide same heterozygosis if all the alleles are equally frequent (Hortl and Clark, 1997) as quantified by effective number of alleles $\left(\mathrm{A}_{\mathrm{e}}\right)$ were obviously more at tetra allelic SSR marker loci than thoseat tri- and bi-allelic markers with a mean of 1.91 alleles (Table 3 ).

\section{Grouping 14 ABL based SSR maker alleles}

The SSR marker alleles-based grouping of 14 ABL into different clusters (Fig. 5) indicated the efficiency of SSR markers to differentiate phenotypically diverse ABL. Thus, higher degree of differences at SSR loci complemented those controlling the quantitative traits. Cross transferable Vigna savi SSR markers differentiated 20 cultivars of Cajanus cajan from 10 genotypes belonging to five wild relatives at $67 \%$ similarity (Agbagwa et al., 2015).

Thus, the present study suggested preferential use of SSR markers from cowpea and soybean and those based on simple di-/trinucleotide repeat motifs for studies designed to examine transferability of cross legume species/genera SSR markers to dolichos bean. The study also indicated the utility of transferable cross legume SSR markers for detection and characterization of polymorphism among ABL differing for pod yield and its component traits.

\section{Acknowledgement}

The senior author acknowledges the award of Senior Research Fellow by Indian Council of Agricultural Research (ICAR), New Delhi for pursuing Ph.D at University of Agricultural Sciences (UAS), Bengaluru, Dr. Lohitashwa and Dr. Fakrudin B. For providing the aliquot of the primers. The results reported in this article are part of the $\mathrm{PhD}$ thesis research.

\section{References}

Agbagwa, I., Datta, S., Patil, P. G., Singh, P. and Nadarajan, N., 2015, Crosstransferable microsatellite markers from Vigna savi are useful for assessing genetic diversity in wild and cultivated Cajanus adams species. Australian J. Crop Sci., 9: 1139-1147. 
Ayyangar, G. N. R. and Nambiar, K. K. K., 1935, Studies in Dolichos lablab (Roxb.) (L.)-The Indian field and garden bean I.Proc Indian Aca Sc., 1: 857-867.

Botstein, D., White, L., Skolick, M. and Davis, R. W., 1980, Construction of a genetic linkage map in man using restriction fragment length polymorphism. American J. Human Genet., 32: 314-331.

Chandra, A., 2011, Use of EST database markers from $M$. truncatula in the transferability to other forage legumes. J. Environ. Biol., 32: 347-354.

Choudhary, S., Sethy, N. K., Bhumika, S. and Bhatia, S., 2009, Development of chickpea EST-SSR markers and analysis of allelic variation across related species. Theor. Appl. Genet., 118:591-608.

Choumane, W., winter, P., Baum, M. and Kahl, G., 2004, Conservation of microsatellite flanking sequences in different taxa of leguminosae. Euphytica, 138: 239-245.

Datta, S., Mahfooz, S., Singh, P., Choudhary, A. K., Chaturvedi, S. K. and Nadarajan, N., 2013, Conservation of microsatellite regions across legume genera increases marker repertoire in pigeonpea. Australian J. Crop Sci., 7: 1990-1997.

Decroocq, V., Fave, M. G., Hagen, L., Bordenave, L. and Decroocq, S., 2003, Development and transferability of apricot and grape EST microsatellite markers across taxa. Theor. Appl. Genet., 106: 912-922.

Doyle, J. J. and Doyle, J. L., 1987, Arapid DNA isolation procedure for small quantities of fresh leaf tissue. Phytochemical Bullet, 19: 11-15.

Gupta, S. and Prasad, M., 2009, Development and characterization of genic SSR markers in Medicago truncatula and their transferability in leguminous and non-leguminous species. Genome, 52: 761-71.

Gupta, S. K., Bansal, R., Vaidya, U. J. and Gopalakrishna, T., 2012, Development of EST-derived microsatellite markers in mungbean [Vigna radiata (L.)Wilczek] and their transferability to other Vigna species. Indian J. Genet., 72: 468-471.

Hartl, D. L. and Clark, A. G., 1997, Principles of Population Genetics. Sinauer, Sunderland, MA.

Humphry, M. E., Konduri, V., Lambrides, C. J., Magner, T. and McIntyre, C. L., 2002, Development of a mung bean (Vigna radiata) RFLP linkage map and its comparison with lablab (Lablab purpureus) reveals a high level of colinearity between the two genomes. Theor. Appl. Genet., 105: 160-166.

Iwata, A., Greenland, C.M., and Jackson, S. A., 2013, Cytogenetics of legumes in the pheseoloid clade, $P$. Genome, 6:18.

Keerthi, C. M., Ramesh, S., Byregowda, M., Mohan Rao, A. and Vaijayanthi, P. V., 2016, High yielding vs. low yielding testers to identify advanced breeding lines for general combining ability in dolichos bean (Lablab purpureus). J. Crop Improv., 30: 95-106.

Kelkar, Y. D., Tyekucheva, S., Chiaromonte, F. and Markova, K. D., 2008, the genome-wide determinants of human and Chimpanzee microsatellite evolution. Genome Res., 18: 30-38.

Magoon, M. L., Singh, A. and Mehra, K. L., 1974, Improved field bean for dry land forage. Indian Farming, 24: 5-7

Nei, M., 1978, Estimation of average heterozygosity and genetic distance from a small number of individuals. Genetics, 89:583-590.

Peakall, R., Gilmore, S., Keys, W., Morgante, M. and Rafalski, A., 1998, Crossspecies amplification of soybean 
(Glycine max) Simple Sequence Repeats (SSRs) within the genus and other legume genera: implications for the transferability of SSRs in plants. Mol. Biol. Evol., 15:1275-1287.

Powell, W. M., Morgante, M., Hudre, C., Hanafey, M. and Vogel, J., 1996, the comparison of RFLP, RAPD, AFLP and SSR (microsatellites) markers for germplasm analysis. Mol. Breed. 2: $225-238$

Raveendar, S., Lee, G. A., Jeon, V. A., Leem, Y. J. and Lee, J. R., 2015, Crossamplification of Vicia sativa and subsp. sativa microsatellites across 22 other Vicia species.Molecules, 20:1543-1550.

She, C. and Jiang, X., 2015, Karyotype analysis of Lablab purpureus (1.) sweet using fluorochrome banding and fluorescence in situ hybridization with rDNA probes. Czech J Genet Plant
Breed, 51:110-116.

Vaijayanthi, P. V., Ramesh, S., Byregowda, M., Mohan Rao, A., Keerthi, C. M., 2016, Identification of traits specific accessions from a core set of dolichos bean germplasm. J. Crop Improv, 30: 244-257.

Yao, L. M., Zhang, L. D., Hu, Y. L., Wang, B. and $\mathrm{Wu}, \mathrm{T}$. L., 2012, Characterization of novel soybean derived simple sequence repeat markers and their transferability in hyacinth bean [Lablab purpureus (L.) Sweet]. Indian J. Genet., 72: 46-53.

Zucchi, M. I., Brondani, R. P. V., Pinheiro, J. B., Chaves, L. J. and Coelho, A. S. G., 2003, Genetic structure and gene flow in Eugenia dysenterica DC in the Brazilian Cerrado utilizing SSR markers. Genet. Mol. Biol., 26:449-457.

\section{How to cite this article:}

Shivakumar, M.S., S. Ramesh, A. Mohan Rao, H.R. Udaykumar and Keerthi, C.M. 2017. Cross Legume Species/Genera Transferability of SSR Markers and their Utility in Assessing Polymorphism among Advanced Breeding Lines in Dolichos Bean (Lablab purpureus L.). Int.J.Curr.Microbiol.App.Sci. 6(8): 656-668. doi: https://doi.org/10.20546/ijcmas.2017.608.083 